\title{
Advances in Scanning Transmission Electron Microscope Cathodoluminescence
}

\author{
S. Meuret ${ }^{1}$, L.H.G Tizei ${ }^{1}$, A. Losquin ${ }^{1}$, R. Bourrellier ${ }^{1}$, L.F. Zagonel ${ }^{1}$, M. Tencé ${ }^{1}$, A. Zobelli ${ }^{1}$, O. \\ Stéphan $^{1}$, M. Kociak ${ }^{1}$ \\ 1. Laboratoire de Physique des Solides, University Paris-Sud, Orsay France
}

The typical sizes at which confinement effects become predominant in optical properties range from few angstroms or nanometer (for excitons) to tens or hundred of nanometers (for plasmons). As in addition the confinement leads to optical properties intimately related to the size and geometry of the objects, it is thus important to have tools able to probe optical, morphological and structural properties at these scales. Optical microscopies and spectroscopies can hardly deliver such spatial resolutions. Recently, electron spectroscopies such as Electron Energy Loss Spectroscopy (EELS) and Cathodoluminescence (CL) used in a Scanning Electron Microscope (STEM) have shown some successes in addressing this issue. We will thus present how recent technical and conceptual developments in EELS and CL have allowed exploring various aspects of plasmonics and quantum optics at the scale relevant for plasmons and quantum emitters.

In order to investigate the physics of plasmons in small metallic nanoparticles, we have probed plasmon modes of the exact same individual particles through combined EELS and CL measurements [1]. CL only probes the radiative modes, in contrast to EELS, which additionally reveals dark modes. The combination of both techniques on the same particles demonstrates that although the radiative modes give rise to very similar spatial distributions when probed by EELS or CL, their resonant energies appear to be different [1]. We trace this phenomenon back to plasmon dissipation. It agrees with electromagnetic numerical simulations and therefore demonstrates that CL and EELS are closely related to optical scattering and extinction, respectively.

Exploring the optical properties of quantum confined objects requires yet another step in instrumentation. Indeed, in those cases, not only the spectral variations arise at scale much smaller than in for plasmons (1 nanometers compared to $10-100 \mathrm{~nm}$ ), but also information on the quantum states of light or the emitters' lifetimes is crucial. We have shown that CL-spectral imaging in the STEM is a very relevant tool to measure and disentangle individual quantum wells emission [2]. A step further has been the introduction of a Hanbury Brown-Twiss intensity interferometer set up fitting our CL-STEM system. This allowed to probe the quantum character of single photon emitters (SPE) with deep subwavelength resolution [3] thanks to the recording of the autocorrelation function $\left(\mathrm{g}^{(2)} \mathrm{CL}(\tau)\right)$. We have applied this method to prove that a well-known defect in h-BN [4] is indeed a SPE [5], making the h-BN a promising platform for quantum nano-optics. In these experiments, the detection of a dip in $\mathrm{g}^{(2)} \mathrm{CL}(\tau)$ at small time delays clearly demonstrated anti-bunching and thus the creation of nonclassical light states. At first sight, this seems like a clear proof of the similarity between CL and photoluminescence (PL) when exciting a unique SPE, as anti-bunching from single photon emitters is a well-known effect in PL. However, we also measured the $\mathrm{g}^{(2)} \mathrm{CL}(\tau)$ resulting from the excitation of several of these defects. In this case, we show that the $\mathrm{g}^{(2)} \mathrm{CL}(\tau)$ of multiple defect centers is dominated by a large nanosecond zero-delay bunching $\left(g^{(2)}(0)>20\right)$, in stark contrast with its flat photoluminescence $\left(\mathrm{g}^{(2)} \mathrm{PL}(\tau)=1\right)$ function. We developed a model showing that this bunching can be attributed to the synchronized emission of several defect centers [6]. We also show that such phenomenon makes it a very promising method of lifetime measurement at the nanometer scale [7].

[1] A. Losquin and al, Nano Letters (2015)

[2] L. Zagonel and al, Nano Letters 11, 568-573 (2011)

[3] L. Tizei and al, PRL 110, 153604 (2013)

[4] L. Museur and al, PRB 78, 155204

[5] R. Bourrellier and al, in preparation 
[6] S. Meuret and al, submitted

[7] S. Meuret and al, in preparation
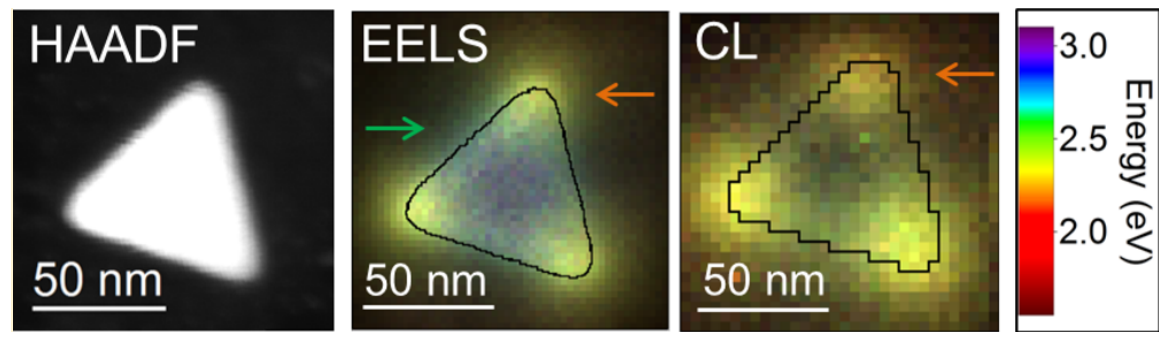

Figure 1. Combined HAADF imaging (top), EELS (middle), and CL (bottom) spatially resolved data sets. Total acquisition times are $157 \mathrm{~s}$ for CL and $16 \mathrm{~s}$ for EELS. In the case of EELS, the spectra are first deconvolved and normalized. The CL and EELS images are generated by coloring each filtered maps of the data sets according to its energy, weighing each pixel of the maps by its intensity and summing all the resulting images. This simplified representation of the EELS and CL data sets straightforwardly shows that the EELS data exhibit both dipolar and higher order modes, whereas the CL data exhibit mainly the dipolar mode. The black lines superimposed on the maps indicate the prism shape as obtained from the HAADF image [1].
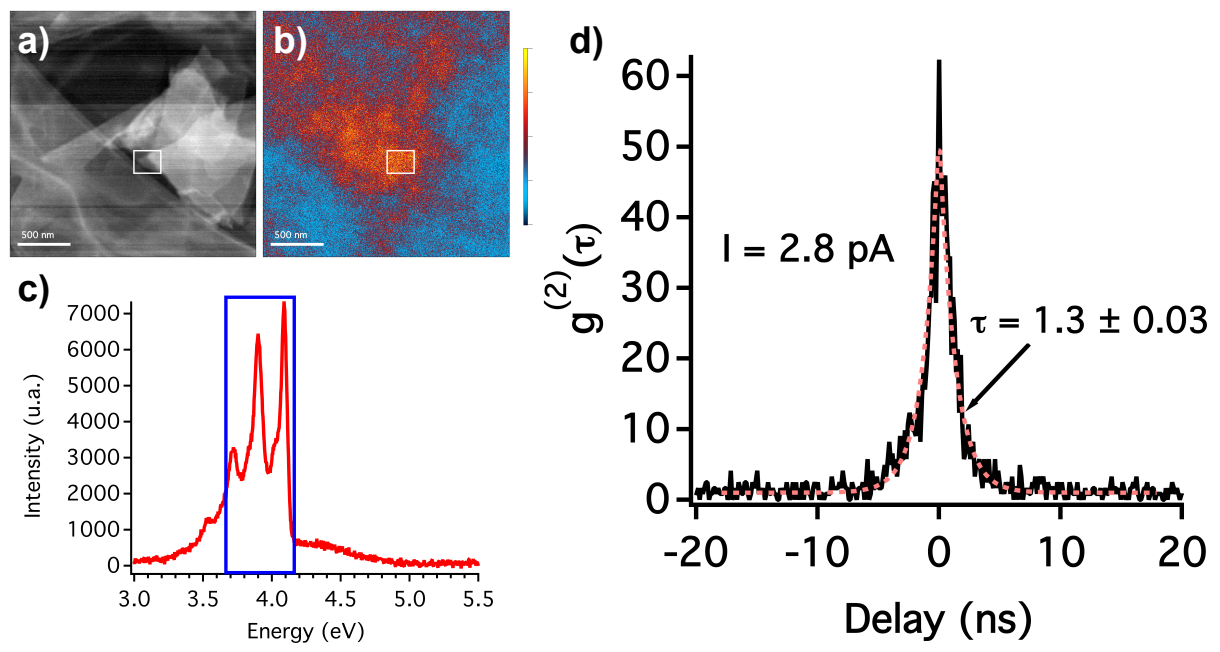

Figure 2. a) HADF and b) emission map of the defects in an h-BN flex. The number of defects excited at the same time is much larger than one. c) Emission spectrum of defects, taken on the white square of the HADF image. The blue square indicates the filter used for the HBT experiment. d) $\mathrm{g}^{(2)}(\tau)$ of the CL signal taken on the white square of the HADF image a). We can see a huge bunching effect $\left(g^{(2)}(0)>50\right)$, for an excitation current of $\mathrm{I}=2.8 \mathrm{pA}$. We retrieve the lifetime of the h-BN defect thought an exponential fit of the $\mathrm{g}^{(2)}(\tau)$ function and find in this case $\tau=1.3 \mathrm{~ns}$ [6]. 